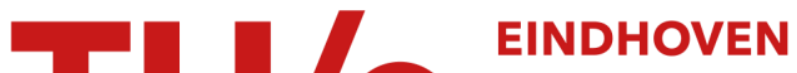 UNIVERSITY OF TECHNOLOGY
}

\section{Infinitely fast diffusion in single-file systems}

\section{Citation for published version (APA):}

Nedea, S. V., Jansen, A. P. J., Lukkien, J. J., \& Hilbers, P. A. J. (2003). Infinitely fast diffusion in single-file systems. Physical Review E - Statistical, Nonlinear, and Soft Matter Physics, 67(4), [046707].

https://doi.org/10.1103/PhysRevE.67.046707

DOI:

10.1103/PhysRevE.67.046707

Document status and date:

Published: 01/01/2003

\section{Document Version:}

Publisher's PDF, also known as Version of Record (includes final page, issue and volume numbers)

\section{Please check the document version of this publication:}

- A submitted manuscript is the version of the article upon submission and before peer-review. There can be important differences between the submitted version and the official published version of record. People interested in the research are advised to contact the author for the final version of the publication, or visit the $\mathrm{DOI}$ to the publisher's website.

- The final author version and the galley proof are versions of the publication after peer review.

- The final published version features the final layout of the paper including the volume, issue and page numbers.

Link to publication

\section{General rights}

Copyright and moral rights for the publications made accessible in the public portal are retained by the authors and/or other copyright owners and it is a condition of accessing publications that users recognise and abide by the legal requirements associated with these rights.

- Users may download and print one copy of any publication from the public portal for the purpose of private study or research.

- You may not further distribute the material or use it for any profit-making activity or commercial gain

- You may freely distribute the URL identifying the publication in the public portal.

If the publication is distributed under the terms of Article $25 \mathrm{fa}$ of the Dutch Copyright Act, indicated by the "Taverne" license above, please follow below link for the End User Agreement:

www.tue.nl/taverne

Take down policy

If you believe that this document breaches copyright please contact us at:

openaccess@tue.nl

providing details and we will investigate your claim. 


\title{
Infinitely fast diffusion in single-file systems
}

\author{
S. V. Nedea, ${ }^{1, *}$ A. P. J. Jansen, ${ }^{2}$ J. J. Lukkien, ${ }^{1}$ and P. A. J. Hilbers ${ }^{1}$ \\ ${ }^{1}$ Department of Mathematics and Computer Science, Technical University of Eindhoven, P.O. Box 513, \\ 5600 MB Eindhoven, The Netherlands \\ ${ }^{2}$ Department of Chemical Engineering, Technical University of Eindhoven, P.O. Box 513, 5600 MB Eindhoven, The Netherlands
}

(Received 30 October 2002; published 23 April 2003)

\begin{abstract}
We have used dynamic Monte Carlo(DMC) methods and analytical techniques to analyze single-file systems for which diffusion is infinitely fast. We have simplified the master equation removing the fast reactions, and we have introduced a DMC algorithm for infinitely fast diffusion. The DMC method for fast diffusion give similar results as the standard DMC with high diffusion rates. We have investigated the influence of characteristic parameters, such as pipe length, adsorption, desorption, and conversion rate constants on the steadystate properties of single-file systems with a reaction, looking at cases when all the sites are reactive and when only some of them are reactive. We find that the effect of fast diffusion on single-file properties of the system is absent even when diffusion is infinitely fast. Diffusion is not important in these systems. Smaller systems are less reactive and the occupancy profiles for infinitely long systems show an exponential behavior.
\end{abstract}

DOI: 10.1103/PhysRevE.67.046707 PACS number(s): 02.70.Uu, 02.60.-x, 05.50.+q, 07.05.Tp

\section{INTRODUCTION}

In one-dimensional systems such as zeolites or other porous structures, diffusion is a very important process. The pores of these structures that have the cross section somewhat larger than a diffusing molecule, are modeled by singlefile systems. In these systems particles move in a concerted fashion, as they are unable to cross each other. This process of single-file diffusion has different characteristics from ordinary diffusion which affects the nature of both transport and conversion by chemical reactions. In Refs. [1] and [2] we have studied the steady-state and transient properties of this system. We have analyzed different situations for diffusion rates, and we have compared the results obtained from simulation and analytical techniques. Often diffusion is a very fast process compared to the other reactions in the system. We are thus interested to be able to model correctly the infinitely fast diffusion. For this purpose, we used dynamic Monte Carlo (DMC) methods [14,16-19] with high regular diffusion rates, assuming that these rates are high enough to model infinitely fast diffusion.

Important work has been done in the area of single-file systems [3-53] and an overview containing comparisons between different results in the field is given in Ref. [1]. In this paper we concentrate on the important properties of infinitely fast diffusion in a single-file system including conversion.

Dynamic Monte Carlo methods for very high rates are not very efficient and the progress of the simulation is slow. Moreover, considering regular reaction rates it is always a problem of making a balance between the diffusion rates high enough so that the infinitely fast diffusion effects are correctly modeled and the performance of the simulation. We derive here a different method to simulate infinitely fast diffusion in single-file systems, starting from the master equation.

The rate equations of some special limiting cases and an

\footnotetext{
*Electronic address: silvia@win.tue.nl
}

analytical description for the productivity of the system are also derived. We study also how the system behavior changes for different sets of kinetic parameters and different distributions of the reactive sites. We categorize also interesting results obtained for profile occupancies for different reactive system and different distribution of the reactive sites.

In Sec. II we specify our mathematical model with the theoretical background for the analytical and simulation results. We introduce the master equation of the systems in Sec. II B and then we simplify the master equation removing the fast reactions in Sec. II C. In Secs. II D 1 and II D 2 we present the simulation methods and we present a DMC algorithm for infinitely fast diffusion. Different analytical results are presented in Sec. III. In Secs. IV A and IV B we analyze different simulation results for the case when all the sites are reactive and when only some of the sites are reactive. We pay special attention to the influence of the length of the pipe and reaction rate constant on the site occupancy of the system.

\section{THEORY}

In this section we will give the theoretical background for our analytical and simulation results. First we will specify our model and we will derive a finite set of exact rate equations starting from the master equation [54]. These rate equations are used in order to derive expressions for the productivity in the system for special cases. We show that we can simplify the master equation describing the evolution of the system over time removing fast reactions. We use a dynamic Monte Carlo method for our simulation results and we give the description of a dynamic Monte Carlo-like algorithm for infinitely fast diffusion.

\section{A. The model}

We model a single-file system by a one-dimensional array (Fig. 1) of sites, each possibly occupied by an adsorbate. 


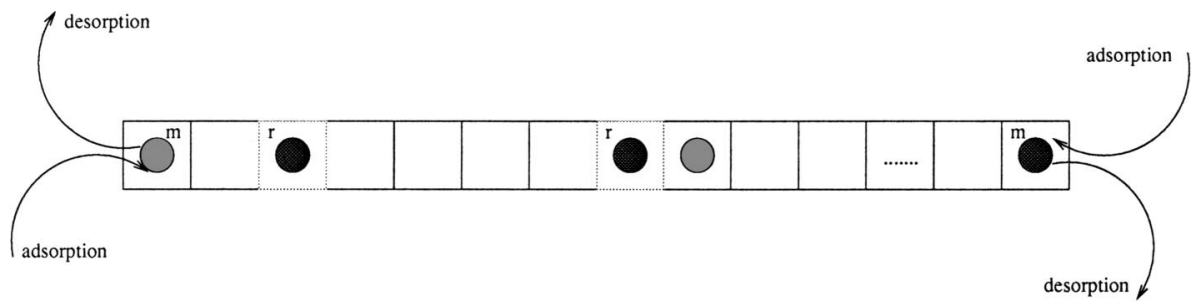

FIG. 1. Picture of a single-file system with two types of adsorbed particles
This is a model for diffusion and reaction in a onedimensional arrangement of particles with hard-core interaction. The sites are numbered $1,2, \ldots, S$. A particle can only move to the right or to the left if an adjacent site is vacant. The sites could be reactive and unreactive and we note with $N_{\text {prot }}$ the number of reactive sites. A reactive site is the only place where a conversion may take place.

We consider two types of adsorbates, $A$ and $B$, in our model and we denote with $Y$ the site occupation of a site, $Y=(*, A, B)$, which stands for an vacant site, a site occupied by $A$, or a site occupied by $B$, respectively. We restrict ourselves to the following monomolecular and bimolecular transitions.

(a) Adsorption and desorption. Adsorption and desorption take place only at the two marginal sites, i.e., the left and rightmost sites at the ends of the system,

$$
\begin{aligned}
& A(\text { gas })+*_{m} \rightarrow A_{m}, \\
& A_{m} \rightarrow A(\text { gas })+*_{m}, \\
& B_{m} \rightarrow B(\text { gas })+*_{m},
\end{aligned}
$$

where subscripts $m$ denotes a marginal site. Note that there is no $B$ adsorption. $B$ s can only be formed by a conversion.

(b) Diffusion. In the pipe, particles are allowed to diffuse via hopping to vacant nearest neighbor sites

$$
\begin{aligned}
& A_{n}+*_{n+1} \leftrightarrow *_{n}+A_{n+1}, \\
& B_{n}+*_{n+1} \leftrightarrow *_{n}+B_{n+1},
\end{aligned}
$$

where the subscripts are site indices: $n=1,2, \ldots, S-1$.

(c) Conversion. An $A$ can transform into a $B$ at a reactive site

$$
A_{r} \rightarrow B_{r}
$$

In the initial state of the system all sites are vacant (no particles in the pipe), since we are interested in the behavior of the system towards equilibrium.

\section{B. Master equation}

Reaction kinetics is described by a stochastic process. Every reaction has a microscopic rate constant associated with it that is the probability per unit time that the reaction occurs. Stochastic models of physical systems can be described by a master equation [54].

By $\alpha, \beta$, we will indicate a particular configuration of the system, i.e., a particular way to distribute adsorbates over all the sites. $P_{\alpha}(t)$ will indicate the probability of finding the system in configuration $\alpha$ at time $t$ and $W_{\alpha \beta}$ is the rate constant of the reaction changing configuration $\beta$ to configuration $\alpha$.

The probability of the system being in configuration $\alpha$ at time $t+d t$ can be expressed as the sum of two terms. The first term is the probability to find the system already in configuration $\alpha$ at time $t$ multiplied by the probability to stay in this configuration during $d t$. The second term is the probability to find the system in some other configuration $\beta$ at time $t$ multiplied by the probability to go from $\beta$ to $\alpha$ during $d t$,

$$
P_{\alpha}(t+d t)=\left(1-d t \sum_{\beta} W_{\beta \alpha}\right) P_{\alpha}(t)+d t \sum_{\beta} W_{\alpha \beta} P_{\beta}(t) .
$$

By taking the limit $d t \rightarrow 0$ this equation reduces to a master equation

$$
\frac{d P_{\alpha}(t)}{d t}=\sum_{\beta}\left[W_{\alpha \beta} P_{\beta}(t)-W_{\beta \alpha} P_{\alpha}(t)\right] .
$$

Analytical results can be derived as follows. The value of a property $X$ is a weighted average over the values $X_{\alpha}$, which is the value of $X$ in configuration $\alpha$,

$$
\langle X\rangle=\sum_{\alpha} P_{\alpha} X_{\alpha}
$$

From this follows the rate equation,

$$
\begin{aligned}
\frac{d\langle X\rangle}{d t} & =\sum_{\alpha} \frac{d P_{\alpha}}{d t} X_{\alpha}=\sum_{\alpha \beta}\left[W_{\alpha \beta} P_{\beta}-W_{\beta \alpha} P_{\alpha}\right] X_{\alpha} \\
& =\sum_{\alpha \beta} W_{\alpha \beta} P_{\beta}\left(X_{\alpha}-X_{\beta}\right) .
\end{aligned}
$$

\section{Master equation for infinitely fast diffusion}

We show that we can simplify the master equation removing the fast reactions. In order to remove fast reactions, we stop distinguishing between configurations that can be transformed into each other by the fast reactions. We split all configurations into disjoint sets such that if $C_{i}$ is one such a set and $\alpha, \beta \in C_{i}$, then $\alpha$ can be transformed into $\beta$, or vice versa by fast reactions. If we denote

$$
\pi_{i}=\sum_{\alpha \in C_{i}} P_{\alpha}
$$

we can derive the master equation for $\pi_{i}$, 


$$
\begin{aligned}
\frac{d \pi_{i}}{d t}= & \sum_{\alpha \in C_{i}} \frac{d P_{\alpha}}{d t}=\sum_{\alpha \in C_{i}} \sum_{\beta}\left[W_{\alpha \beta} P_{\beta}-W_{\beta \alpha} P_{\alpha}\right] \\
= & \sum_{\alpha \in C_{i}} \sum_{j} \sum_{\beta \in C_{j}}\left[W_{\alpha \beta} P_{\beta}-W_{\beta \alpha} P_{\alpha}\right] \\
= & \sum_{j}\left(\sum_{\alpha \in C_{i}} \sum_{\beta \in C_{j}} W_{\alpha \beta} \frac{P_{\beta}}{\pi_{j}}\right) \pi_{j} \\
& -\sum_{j}\left(\sum_{\beta \in C_{j}} \sum_{\alpha \in C_{i}} W_{\beta \alpha} \frac{P_{\alpha}}{\pi_{i}}\right) \pi_{i}=\sum_{j}\left[\omega_{i j} \pi_{j}-\omega_{j i} \pi_{i}\right]
\end{aligned}
$$

with $\omega_{i j}=\Sigma_{\alpha \in C_{i}} \sum_{\beta \in C_{j}} W_{\alpha \beta} P_{\beta} / \pi_{j}$.

We see that all fast reactions have disappeared; they only contribute to $\omega_{i i}$, which can be left out of the ME (master equation). In order to calculate $\omega_{i j}$, we need the conditional probabilities $P_{\beta} / \pi_{j}$. Because we have fast reactions connecting the $\beta \mathrm{s}$ in $C_{j}$ we may assume that these $\beta \mathrm{s}$ are in steady state with respect to each other. Hence, the conditional probability $P_{\beta} / \pi_{j}$ is nothing but the probability of $\beta$ in steady-state if we restrict ourselves to the configurations in $C_{j}$.

\section{Simulation methods}

\section{Dynamic Monte Carlo}

DMC methods allow us to simulate the system governed by the master equation over time. We simplify the notation of the master equation by defining a matrix $\mathbf{W}$ containing the rate constants $W_{\alpha \beta}$, and a diagonal matrix $\mathbf{R}$ by $R_{\alpha \beta}$ $\equiv \Sigma_{\gamma} W_{\gamma \beta}$, if $\alpha=\beta$, and 0 otherwise. If we put the probabilities of the configurations $P_{\alpha}$ in a vector $\mathbf{P}$, we can write the master equation as

$$
\frac{d \mathbf{P}}{d t}=-(\mathbf{R}-\mathbf{W}) \mathbf{P},
$$

where $\mathbf{R}$ and $\mathbf{W}$ are time independent. We also introduce a new matrix $\mathbf{Q}, \mathbf{Q}(t) \equiv \exp [-\mathbf{R} t]$.

This matrix is time dependent by definition, and we can rewrite the master equation in the integral form

$$
\mathbf{P}(t)=\mathbf{Q}(t) \mathbf{P}(0)+\int_{0}^{t} d t^{\prime} \mathbf{Q}\left(t-t^{\prime}\right) \mathbf{W P}\left(t^{\prime}\right) .
$$

By substitution we get from the right-hand side for $P\left(t^{\prime}\right)$,

$$
\begin{aligned}
\mathbf{P}(t)= & {\left[\mathbf{Q}(t)+\int_{0}^{t} d t^{\prime} \mathbf{Q}\left(t-t^{\prime}\right) \mathbf{W} \mathbf{Q}\left(t^{\prime}\right)+\int_{0}^{t} d t^{\prime} \int_{0}^{t^{\prime}} d t^{\prime \prime}\right.} \\
& \left.\times \mathbf{Q}\left(t-t^{\prime}\right) \mathbf{W} \mathbf{Q}\left(t^{\prime}-t^{\prime \prime}\right) \mathbf{W} \mathbf{Q}\left(t^{\prime \prime}\right)+\cdots\right] \mathbf{P}(0)
\end{aligned}
$$

Suppose at $t=0$ the system is in configuration $\alpha$ with probability $P_{\alpha}(0)$. The probability that, at time $t$, the system is still in configuration $\alpha$ is given by $Q_{\alpha \alpha}(t) P_{\alpha}(0)$ $=\exp \left(-R_{\alpha \alpha} t\right) P_{\alpha}(0)$. This shows that the first term represents the contribution to the probabilities when no reaction takes place up to time $t$. The matrix $\mathbf{W}$ determines how the probabilities change when a reaction takes place. The second term represents the contribution to the probabilities when no reaction takes place between times 0 and $t^{\prime}$, some reaction takes place at time $t^{\prime}$, and then no reaction takes place between $t^{\prime}$ and $t$. The subsequent terms represent contributions when two, three, four, etc., reactions take place. The idea of the DMC method is not to compute probabilities $P_{\alpha}(t)$ explicitly, but to start with some particular configuration, representative for the initial state of the experiment one wants to simulate, and then generate a sequence of other configurations with the correct probability. The method generates a time $t^{\prime}$ when the first reaction occurs according to the probability distribution $1-\exp \left[-R_{\alpha \alpha} t\right]$. At time $t^{\prime}$ a reaction takes place such that a new configuration $\alpha^{\prime}$ is generated by picking it out of all possible new configurations $\beta$ with a probability proportional to $W_{\alpha^{\prime} \alpha}$. At this point we can proceed by repeating the previous steps, drawing again a time for a new reaction and a new configuration $[55,56]$. One of the most popular DMC method in the literature is random selection method(RSM) [55]. We use this method to simulate the master equation of our system.

\section{A dynamic Monte Carlo algorithm for infinitely fast diffusion}

In Sec. $\mathrm{C}$ we have derived the ME distinguishing between configurations that can be transformed into each other by fast reactions. Starting from $\mathrm{ME}(5)$ we give a DMC algorithm for simulating infinitely fast diffusion. For our model, diffusion is much faster than adsorption and desorption, so the sets are all configurations that are connected by diffusion. These sets can be labeled only by the sequences of particles $A$ and $B$, as only the number of $A$ and $B$ particles and their order in the pipe is important to distinguish the configurations within a set. Moreover, all probabilities of configurations within a set are the same. This means that $\pi_{j} / P_{\beta}$ is the number of configurations in $C_{j}$. There are $\left(\begin{array}{l}S \\ n\end{array}\right)$ ways to distribute $n$ particles over $S$ sites. We have then

$$
\frac{P_{\beta}}{\pi_{n}}=\left(\begin{array}{l}
S \\
n
\end{array}\right)^{-1}
$$

with $P_{\beta} \in C_{n}$. The summation $\sum_{\beta \in C_{n}}$ sums over $\left(\begin{array}{l}S \\ n\end{array}\right)$ configurations. However, for adsorption the left-most or the right-most site should be vacant. This gives us $\left(\begin{array}{c}S-1 \\ n\end{array}\right)$ possible configurations. Each of these configurations gives just one configuration in the summation over $\alpha$. So we get

$$
\omega_{a d s}=2 W_{a d s} \frac{\left(\begin{array}{c}
S-1 \\
n
\end{array}\right)}{\left(\begin{array}{l}
S \\
n
\end{array}\right)}=2 W_{\text {ads }} \frac{S-n}{S}=2 W_{a d s}(1-\theta),
$$

where $\omega_{a d s}$ is the transition probability for the transition from a configuration within the set with $n$ particles to a configuration within the set with $n+1$ particles. Similarly, we find that 


$$
\omega_{d e s}=2 W_{d e s} \frac{\left(\begin{array}{c}
S-1 \\
n-1
\end{array}\right)}{\left(\begin{array}{l}
S \\
n
\end{array}\right)}=\frac{2 W_{d e s} n}{S}=2 W_{d e s} \theta
$$

In both expressions $\theta=n / S$ is the coverage.

The dynamic Monte Carlo (DMC) algorithm that we have used to simulate the system consists of the following steps.

(1) Compute the time for the next adsorption or desorption. If the current time is $t$ then the time for that process is $t+\Delta t$ with

$$
\Delta t=-\frac{\ln r}{2(1-\theta) W_{\mathrm{ads}}+2 \theta W_{\mathrm{des}}},
$$

where $r$ is a random number picked from the uniform distribution on the interval $\langle 0,1]$ and $\theta$ is the probability that the marginal site is occupied. With infinitely fast diffusion this probability is given by $\theta=\left(N_{A}+N_{B}\right) / S$ with $N_{\mathrm{A}}$ and $N_{\mathrm{B}}$ the number of $A$ 's and $B$ 's respectively in the system.

(2) Compute for each $A$ in the system a time when it will transform into a $B$. This time is given by $t+\tau$ with

$$
\tau=-\frac{\ln r}{P W_{\mathrm{rx}}}
$$

In this expression $P$ stands for the probability that the $A$ is at a protonic site. If we number the particles in the system from left to right $1,2, \ldots, N_{A}+N_{B}$, and the sites also from left to right $1,2, \ldots, S$ then the probability that particle number $n$ is at site number $s, P_{n}^{s}$, is given by

$$
P_{n}^{s}=\frac{\left(\begin{array}{c}
s-1 \\
n-1
\end{array}\right)\left(\begin{array}{c}
S-s \\
N_{A}+N_{B}-n
\end{array}\right)}{\left(\begin{array}{c}
S \\
N_{A}+N_{B}
\end{array}\right)} .
$$

$P$ for particle $n$ is then the sum of this expression over all protonic sites

$$
P=\sum_{s=1}^{S} P_{n}^{s} \delta_{s}
$$

where $\delta_{s}=1$ if site $s$ is protonic.

(3) Change those $A \mathrm{~s}$ with $\tau<\Delta t$ into $B \mathrm{~s}$.

(4) Determine the next process at the marginal sites. It is an adsorption with probability proportional to $(1-\theta) W_{\text {ads }}$ and a desorption with probability proportional to $\theta W_{\text {des }}$. The process is equally likely to occur on the left- or the righthand side.

(5) Change the number of particles in the system according to the next process at the marginal sites.

(6) Update the time.

(7) Repeat steps $1-6$.

A validation of this method can be found in Ref. [57].

\section{ANALYTICAL RESULTS}

In this section, for some special cases such as low loading limit and fast and slow reactions, we are able to derive some expressions for the productivity in the steady state. For the case of low loading limit we can also derive the rate equations of the system.

\section{A. The low loading limit.}

In this case we can assume that there is never more than one particle in the system. The following rate equations then hold:

$$
\begin{gathered}
\frac{d\langle A\rangle}{d t}=\frac{2 W_{\mathrm{ads}}}{S}\langle *\rangle-\frac{2 W_{\mathrm{des}}}{S}\langle A\rangle-\frac{N_{\mathrm{prot}} W_{\mathrm{rx}}}{S}\langle A\rangle, \\
\frac{d\langle B\rangle}{d t}=-\frac{2 W_{\mathrm{des}}}{S}\langle B\rangle+\frac{N_{\mathrm{prot}} W_{\mathrm{rx}}}{S}\langle A\rangle,
\end{gathered}
$$

where $N_{\text {prot }}$ is the number of reactive sites in the system. In the rate equations for $A$, the contributions to the probability to have an $A$ in the system is given by the adsorption to an open end, while the loss to this probability is given by the desorption to an open end and also by the conversion. The probability to have an adsorption of a particle $A$ into the system equals the adsorption rate constant $W_{\text {ads }}$ times the probability to have a site vacant $(\langle *\rangle)$ times the probability to be at one of the marginal sites $(2 / S)$. The probability to have a desorption of an $A$ equals the desorption rate constant $W_{\text {des }}$ times the probability to have an $A$ in the system $\langle A\rangle$ times the probability to be at one of the marginal sites $(2 / S)$. The last term stands for the probability of a reaction of $A$ into a $B$ and this equals the probability to have an $A$ in the system $(\langle A\rangle)$ times the reaction rate constant $W_{\mathrm{rx}}$ times the probability to be at one reactive site $\left(N_{\text {prot }} / S\right)$. In the same way we derive the rate equations for $B$.

Here $\langle X\rangle$ is the probability that there is an $X$ at an arbitrary site. For steady state we get

$$
\begin{aligned}
& \langle A\rangle=\frac{2 W_{\mathrm{ads}} W_{\mathrm{des}}}{\left(W_{\mathrm{ads}}+W_{\mathrm{des}}\right)\left(2 W_{\mathrm{des}}+N_{\mathrm{prot}} W_{\mathrm{rx}}\right)}, \\
& \langle B\rangle=\frac{N_{\mathrm{prot}} W_{\mathrm{ads}} W_{\mathrm{rx}}}{\left(W_{\mathrm{ads}}+W_{\mathrm{des}}\right)\left(2 W_{\mathrm{des}}+N_{\mathrm{prot}} W_{\mathrm{rx}}\right)} .
\end{aligned}
$$

From this equation we immediately get the turnover frequency defined as the rate of $B$ desorption per reactive site

$$
\omega_{\mathrm{TOF}}=\frac{2 W_{\mathrm{des}}\langle B\rangle}{N_{\mathrm{prot}}}=\frac{2 W_{\mathrm{des}}(1-\langle A\rangle)}{N_{\mathrm{prot}}} .
$$

If we replace the steady-state expressions for $A$ and $B$ in this equation we get

$$
\omega_{\mathrm{TOF}}=\frac{2 W_{\mathrm{ads}} W_{\mathrm{des}} W_{\mathrm{rx}}}{\left(W_{\mathrm{ads}}+W_{\mathrm{des}}\right)\left(2 W_{\mathrm{des}}+N_{\mathrm{prot}} W_{\mathrm{rx}}\right)} .
$$

We see that in this limit the turnover frequency does not depend on the system size. 
Comparing the number of $B$ s produced from the analytical results with the DMC results for the case when $W_{\text {ads }}$ $=0.0033, W_{\mathrm{des}}=0.9967, W_{\mathrm{rx}}=0.1$, and different distributions of the reactive sites, we remark that we get similar results.

\section{B. Fast and slow $A \rightarrow B$ reaction}

If the reaction is fast, and there are not too many particles in the system, then all particles in the system are $B$ s. This means

$$
\langle B\rangle=\frac{W_{\mathrm{ads}}}{W_{\mathrm{ads}}+W_{\mathrm{des}}}
$$

and

$$
\omega_{\mathrm{TOF}}=\frac{1}{N_{\mathrm{prot}}} \frac{2 W_{\mathrm{ads}} W_{\mathrm{des}}}{W_{\mathrm{ads}}+W_{\mathrm{des}}} .
$$

The restriction of not too many particles is necessary, because all particles should always be able to reach a protonic site. This means that

$$
\frac{W_{\text {ads }}}{W_{\text {ads }}+W_{\text {des }}} \ll \frac{N_{\text {inert }}}{S}
$$

must hold, where $N_{\text {inert }}$ is the distance between the marginal site and the first protonic site. This relation depends on the distribution of the reactive sites. When reaction is fast this means that it depends on the distance from the margins to the first protonic site, $N_{\text {inert }}$.

Comparing the site occupancy with $B \mathrm{~s}(19)$ with results from the DMC simulations, we find that for the reaction rate constants $W_{\text {ads }}=0.03333, W_{\text {des }}=0.96667, W_{\mathrm{rx}}=10$ and all the sites reactive, the results are similar.

If the reaction is slow, then there are only occasionally $B \mathrm{~s}$ in the system. This means that

$$
\langle A\rangle=\frac{W_{\text {ads }}}{W_{\text {ads }}+W_{\text {des }}} .
$$

All particles in the system will be renewed between two subsequent formations of a $B$. Therefore

$$
\omega_{\mathrm{TOF}}=\frac{W_{\mathrm{ads}} W_{\mathrm{rx}}}{W_{\mathrm{ads}}+W_{\mathrm{des}}} .
$$

Comparing the site occupancy with $A \mathrm{~s}(22)$ with results from the DMC simulations, we find that for the reaction rate constants $W_{\text {ads }}=0.03333, W_{\text {des }}=0.96667, W_{\text {rx }}=0.001$ and all the sites reactive, the results are similar.

\section{SIMULATION RESULTS AND DISCUSSION}

\section{A. All sites reactive}

We remark that DMC methods with regular high rates for diffusion tend to give similar results as DMC method for infinitely fast diffusion described in Sec. II D 2. The results of these comparisons are in Fig. 2. We conclude that for

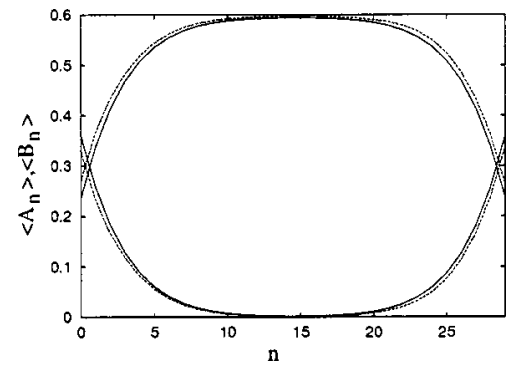

FIG. 2. Profile occupancies for a system of length $S=30, W_{\text {ads }}$ $=0.6, W_{\mathrm{des}}=0.4$, and $W_{\mathrm{rx}}=0.1$. The continous lines are the profile occupancies for $A$ (the lower) and $B$ (the higher) using DMC for infinitely fast diffusion. The dotted lines are the profile occupancies for $A$ (the lower) and $B$ (the higher) using DMC with a regular high rate for diffusion $\left(W_{\text {diff }}=1600\right)$.

infinitely fast diffusion the DMC method we have defined is a correct method to simulate the behavior of the system in the limit $W_{\text {diff }} \longrightarrow \infty$.

In Ref. [1] we have seen that for the case when all the sites are reactive, the site occupancies of the system obtained from DMC simulations show that the system is not homogeneous even for very fast diffusion rates. We find the same effect also using DMC for infinitely fast diffusion, for different loadings and for different reaction rate constants.

We study also the dependence of the occupancy profiles on the reaction rate constant at different loadings of the system.

The simulation results in Fig. 3 show how the shape of the profiles changes with reaction rate $W_{\text {rx }}$ for high and low loading of the pipe, when all the sites are reactive. We find that for high loadings, as an effect of the blocking, the middle sites have the same probability to be occupied for both fast and slow reactive systems. Only the occupancy of marginal sites is influenced by the reactivity, such that in fast reactive systems we have a higher probability to have a $B$
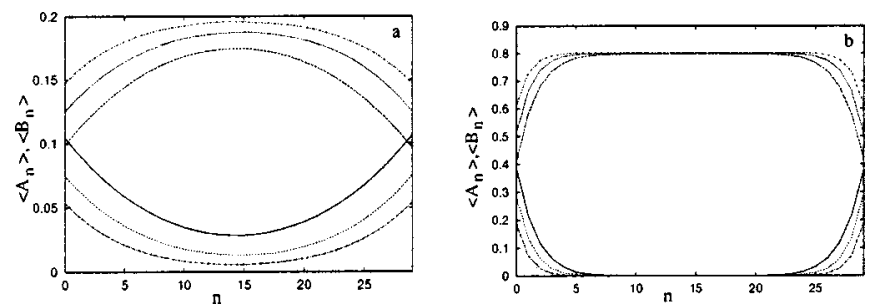

FIG. 3. (a) Dynamic Monte Carlo results for site occupancy for the case of infinitely fast diffusion and low loading ( $W_{\mathrm{ads}}=0.2$, $W_{\text {des }}=0.8$ ) of a system of length $S=30$. The continuous line is for the site occupancy for $W_{\mathrm{rx}}=0.1$, the first dotted line near the continous line is for $W_{\mathrm{rx}}=0.2$, and the second is for $W_{\mathrm{rx}}=0.4$. (b) Dynamic Monte Carlo results for site occupancy for the case of infinitely fast diffusion and high loading ( $\left.W_{\mathrm{ads}}=0.8, W_{\mathrm{des}}=0.2\right)$ of a system of length $S=30$. The continuous line is for the site occupancy for $W_{\mathrm{rx}}=0.1$, the first dotted line near the continous line is for $W_{\mathrm{rx}}=0.2$, and the second is for $W_{\mathrm{rx}}=0.4$. In (a) and (b) the lines at high occupancies correspond to $\left\langle B_{n}\right\rangle$ and at low occupancies correspond to $\left\langle A_{n}\right\rangle$. 


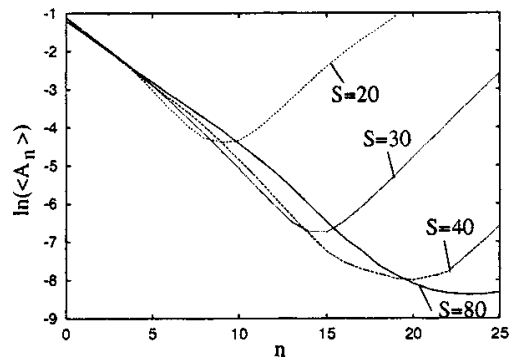

FIG. 4. The logarithm of the DMC(random selection method) profile occupancies $\left(\left\langle A_{n}\right\rangle\right)$ for $W_{\mathrm{ads}}=0.6, W_{\mathrm{des}}=0.4, W_{\mathrm{rx}}=0.1$, and when all the sites are reactive, for various system sizes $S$.

near the marginal sites, and, in consequence, a better productivity. For slow reactive systems, the occupancy profiles are scaled with reaction rate constant. We notice that the productivity is growing, increasing the reaction rate in case of low loading systems almost as fast as in the case of the high loading systems because of the diminished effect of the blocking in the pipe. Comparing, for instance, the rate of growth for $B$ production when reaction rate constant is increasing from $W_{r x}=0.1 \quad$ to $\quad W_{r x}=0.4 \quad\left(B_{\text {prod }}^{W_{r x}=0.4}\right.$ $\left.-B_{\text {prod }}^{W_{r x}=0.1}\right) / S$, in case of low loading $(\theta=0.2)$ and high loading ( $\theta=0.8$ ), we find almost the same rate of growth in both the cases, and that is 0.5 .

In Fig. 4 we have the logarithmic shape of the occupancy profiles for $A$ and $B$. These profiles show that smaller systems are less reactive as less $A$ s are inside the pipe. This explains the faster decrease of $\left\langle A_{n}\right\rangle$ for small systems in Fig. 4. For infinitely long systems we expect to have straight lines corresponding to an exponential decrease of $\left\langle A_{n}\right\rangle$.

\section{B. Only some of the sites reactive}

For all the sites reactive, in paper [1], we have shown that even when $W_{\text {diff }} \rightarrow \infty$, DMC results with regular high diffu- sion rates indicate that the system does not become homogeneous. Using DMC for infinitely fast diffusion we find that also for different distribution of the reactive sites we find a nonhomogeneous distribution of the particles in the system. In Fig. 5 we can see that for marginal sites reactive and for middle sites reactive as well, we have single-file effects also for infinitely-fast diffusion for different rates of reactions.

We notice that the profiles in case of high loadings are very slowly dependent on conversion, both for middle and for marginal sites reactive. Comparing with simulation results for occupancy profiles in Ref. [1], where middle sites and marginal sites are reactive, and for regular diffusion rate constants in the domain $(2 \ldots 10)$, we notice that the profiles are similar. We can conclude that since the effect of infinitely fast diffusion is absent for single-file systems, the diffusion is not so important in these systems.

For low loading, when marginal sites are reactive, the occupancy profiles are scaled with $W_{\mathrm{rx}}$. We notice that conversion in Fig. 5 is the rate determining step. In this case, the middle sites does not have the same occupancy for different reaction rate constants like in the case of all the sites reactive, but are strongly dependent on $W_{r x}$.

We notice also that the productivity in case marginal sites are reactive is growing faster, increasing the reaction rate constants at low loadings than at high loadings. Comparing, for instance, the rate of growth for $B$ production $B_{\text {prod }}$ when reaction rate constant is increasing from $W_{r x}=0.1$ to $W_{r x}$ $=0.4$, in case of low loading $(\theta=0.2)$ and high loading $(\theta$ $=0.8$ ), we find that the rate of growth of $B$ productivity at low loadings $(0.3)$ is larger than at high loadings $(0.25)$.

When we have middle sites reactive we have higher probability to find $A$ s near marginal sites. The productivity is smaller than in all other cases. The profiles are again scaled with $W_{\mathrm{rx}}$ for low loadings. For high loadings and middle sites reactive, the profiles for different conversion rates are almost similar, so the productivity can only be increased in this case by increasing the number of reactive sites.
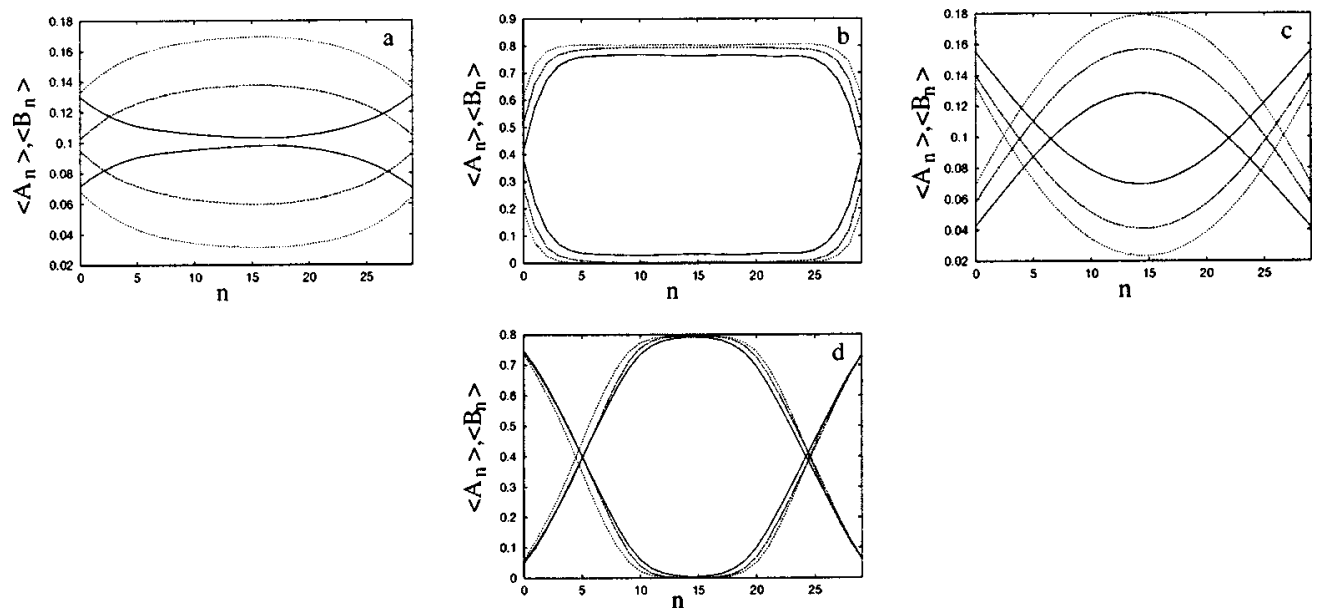

FIG. 5. Dynamic Monte Carlo results for site occupancy for the case of infinitely fast diffusion. The first two (a) and (b) are for the case of 5 marginal sites reactive at each end and the last two (c) and (d) for the case of 10 middle sites reactive. The first figure is for the case of low loading ( $W_{\mathrm{ads}}=0.2, W_{\mathrm{des}}=0.8$ ), and the second is for high loading ( $\left.W_{\mathrm{ads}}=0.8, W_{\mathrm{des}}=0.2\right)$ at different reaction rates $\left(W_{\mathrm{rx}}=0.1,0.2\right.$, 0.4). The third and the fourth figures are for the same parameters as the first and the second, but for the case of 10 middle sites reactive. 


\section{SUMMARY}

We have used DMC methods and analytical techniques to analyze single-file systems for which diffusion is infinitely fast. We simplified the ME removing fast diffusion, and we have presented a DMC algorithm for infinitely fast diffusion that simulate this ME. We show that DMC with regular high rates gives the same results as DMC with infinitely fast diffusion. The fundamental assumption considered for infinitely fast diffusion in the analytical results is that all configurations related by diffusion have the same probability.

In the limiting cases such as low loading limit and slow and fast conversions, we are able to derive expressions for the $B$ productivity. We notice that the number of $B$ s produced per unit time in these cases do not depend on the system size. Comparisons between analytical and DMC results reveal similar results for the productivity.

DMC results show that when all the sites are reactive and when only some of the sites are reactive, diffusion has no influence on the single-file properties of the system. Different results for the dependencies of the occupancy profiles and productivity on the reaction rate constant and different distributions are categorized. The occupancy profiles show that smaller systems are less reactive since less $A$ s are inside the pipe.
[1] S.V. Nedea, A.P.J. Jansen, J.J. Lukkien, and P.A.J. Hilbers, Phys. Rev. E 65, 066701 (2002).

[2] S.V. Nedea, A.P.J. Jansen, J.J. Lukkien, and P.A.J. Hilbers, Phys. Rev. E 66, 066705 (2002).

[3] D. Paschek and R. Krishna, Phys. Chem. Chem. Phys. 3, 3185 (2001).

[4] D. Paschek and R. Krishna, Phys. Chem. Chem. Phys. 2, 2389 (2000).

[5] C. Rödenbeck, J. Kärger, and K. Hahn, J. Catal. 157, 656 (1995).

[6] P.H. Nelson and S.M. Auerbach, J. Chem. Phys. 18, 110 (1999).

[7] S.M. Auerbach and H.I. Metiu, J. Chem. Phys. 106, 2893 (1996).

[8] C. Saravanan and S.M. Auerbach, J. Chem. Phys. 110, 11000 (1999)

[9] C. Saravanan and S.M. Auerbach, J. Chem. Phys. 107, 8132 (1997).

[10] M.-O. Coppens, A. Bell, and Chakraborty, Chem. Eng. Sci. 54, 3455 (1999).

[11] M.-O. Coppens, A. Bell, and Chakraborty, Chem. Eng. Sci. 53, 2053 (1998).

[12] M.-O. Coppens, A. Bell, and Chakraborty, Scientific Computing in Chemical Engineering, edited by F. Keil, W. Mackens, H. Voss, and J. Werther (Springer-Verlag, Berlin, 1999), pp. 200-207.

[13] F.J. Keil, R. Krishna, and M.-O. Coppens, Rev. Chem. Eng. 16, 71 (2000).

[14] R.J. Gelten, R. van Santen, and A.P.J. Jansen, in Molecular Dynamics: From classical to Quantum Methods, edited by P.B. Balthuena and J.M. Seminara (Elsevier, Amsterdam, 1999).

[15] H. Mamada and F. Takano, J. Phys. Soc. Jpn. 25, 675 (1968).

[16] R.J. Gelten, R. Santen, and A.P.J. Jansen, Isr. J. Chem. 38, 415 (1998).

[17] A.P.J. Jansen, Comput. Phys. Commun. 86, 1 (1995).

[18] J. Segers, Algorithms for the Simulation of Surface Processes (Technische Universiteit Eindhoven, Eindhoven, 1999).

[19] K. Binder, Monte Carlo Methods in Statistical Physics (Springer, Berlin, 1986).

[20] D. Gillespie, J. Comput. Phys. 22, 403 (1976).

[21] D. Gillespie, J. Phys. Chem. 81, 2340 (1977).
[22] J. Mai, V. Kuzovkov, and W. Niessen, J. Chem. Phys. 100, 6073 (1994).

[23] J. Mai, V. Kuzovkov, and W. Niessen, J. Chem. Phys. 100, 8522 (1994).

[24] R.J. Baxter, Exactly Solved Models in Statistical Mechanics (Academic Press, New York, 1982).

[25] M. Smoluchowski, Z. Phys. Chem., Stoechiom. Verwandtschaftsl. 92, 129 (1917).

[26] A.J. Guttmann, Asymptotic Analysis of Power-Series Expansions in Phase Transitions and Critical Phenomena (Academic Press, New York 1989).

[27] D.J. Amit, Field theory, the Renormalization Group, and Critical Phenomena (World Scientific, Singapore, 1984).

[28] T.M. Liggett, Interacting Particle Systems (Springer, Berlin, 1985).

[29] H. Spohn, Interacting Particle Systems (Springer, Berlin, 1991).

[30] B.M. McCoy and T.T. Wu, The Two-Dimensional Ising Model (Harvard University Press, Cambridge, 1973).

[31] A.M. Polyakov, Pis'ma Zh. Eksp. Teor. Fiz. 12, 538 (1970) [Sov. Phys. JETP 12, 381 (1970)].

[32] J.L. Cardy, Phase Transitions and Critical Phenomena (Academic Press, New York, 1987).

[33] M. Henkel, Conformal Invariance and Critical Phenomena (Springer Verlag, Berlin, 1999).

[34] B. Schmittmann and R.K.P. Zia, Statistical Mechanics of Driven Diffusive Systems in Phase Transitions and Critical Phenomena (Academic Press, New York, 1995).

[35] J. Marro and R. Dickman, Nonequilibrium Phase Transitions in Lattice Models (Cambridge University Press, Cambridge, 1998).

[36] F.C. Alcaraz, M.H.M. Droz, and V. Rittenberg, Ann. Phys. (N.Y.) 230, 250 (1994).

[37] B. Derrida and M. Evans, in Nonequilibrium Statistical Mechanics in One Dimension (Cambridge University Press, London, 1997).

[38] B. Derrida and M.R. Evans, J. Phys. A 32, 4833 (1999).

[39] B. Derrida, V. Hakim, and R. Zeitak, Phys. Rev. Lett. 77, 2871 (1996).

[40] Ben-Naim and P.L. Krapivsky, J. Phys. A 27, 481 (1994).

[41] V. Privman, Nonequilibrium Statistical Mechanics in One Dimension (Cambridge University Press, London, 1997). 
[42] M.R. Evans, N. Rajewsky, and E.R. Speer, J. Stat. Phys. 95, 45 (1999).

[43] L.H. Gwa and H. Spohn, Phys. Rev. A 46, 844 (1992).

[44] F.C. Alcaraz, Int. J. Mod. Phys. B 8, 3349 (1994).

[45] C.R. Doering, M.A. Burschka, and W. Horsthemke, J. Stat. Phys. 65, 953 (1991).

[46] B. Derrida, E. Domany, and D. Mukamel, J. Stat. Phys. 69, 667 (1992).

[47] G. Schutz and E. Domany, J. Stat. Phys. 72, 277 (1993).

[48] B. Derrida, M.R. Evans, V. Hakim, and V. Pasquier, J. Phys. A 26, 1493 (1993).

[49] J.G. Tsikoyannis and J. Wei, Chem. Eng. Sci. 46, 233 (1991).

[50] C. Rödenbeck, J. Kärger, and K. Hahn, Phys. Rev. E 55, 5697 (1997).
[51] M.S. Okino, R.Q. Snurr, H.H. Kung, J.E. Ochs, and M.L. Mavrovouniotis, J. Chem. Phys. 111, 2210 (1999).

[52] R. Dickman and I. Jensen, Phys. Rev. Lett. 67, 2391 (1991).

[53] G. Grinstein, Z.W. Lai, and D.A. Browne, Phys. Rev. A 40, 4820 (1989).

[54] V. Kampen, Stochastic Processes in Physics and Chemistry (Elsevier Science Publishers B.V., Amsterdam, 1981).

[55] J. Lukkien, J. Segers, P.A.J. Hilbers, R.J. Gelten, and A.P.J. Jansen, Phys. Rev. E 58, 2598 (1998).

[56] R.J. Gelten, A.P.J. Jansen, R. Santen, J.J. Lukkien, and P. Hilbers, J. Chem. Phys. 108, 5921 (1998).

[57] S.V. Nedea, Analysis and Simulations of Catalytic Reactions (Technische Universiteit Eindhoven, Eindhoven, 2003). 